\title{
Correlation between Inflammatory Markers and Liver Enzymes in Patients with Post-Traumatic Stress Disorder
}

\author{
Almir Fajkic ${ }^{1}$, Jasmin Musanovic², Orhan Lepara ${ }^{3}$, Miralem Music ${ }^{4}$, \\ Esad Pepic ${ }^{5}$, Alen Dzubur 6 , Amela Dzubur ${ }^{7}$, Delila Lisica ${ }^{8}$ \\ 1, 4,5 Department of Pathophysiology, University of Sarajevo, Sarajevo, Bosnia and Herzegovina. ${ }^{2}$ Department \\ of Biology and Human Genetics, University of Sarajevo, Bosnia and Herzegovina. ${ }^{3}$ Department of Human \\ Physiology, University of Sarajevo, Sarajevo, Bosnia and Herzegovina. ${ }^{6}$ Department of Cardiology, Clinic for \\ Heart, Blood Vessel and Rheumatic Diseases, Clinical Centre University of Sarajevo, Sarajevo, Bosnia and \\ Herzegovina. ${ }^{7,8}$ Department of Public Health, University of Sarajevo, Sarajevo, Bosnia and Herzegovina.
}

\section{ABSTRACT}

\section{BACKGROUND}

Post-traumatic stress disorder (PTSD) is a mental health condition that is triggered by a terrifying event either experiencing it or witnessing it. Although the pathogenesis is still unknown, some researches indicate inflammatory background and liver dysfunction as a part of the disease. We wanted to determine inflammatory markers' levels and investigate the correlation with liver enzymes in PTSD patients.

\section{METHODS}

This cross-sectional study included 60 male subjects aged between 40 - 60 years. Subjects were divided into two groups: a group of veterans with combat exposure and PTSD according to DSM-IV criteria and a control group of healthy subjects without combat exposure. WBC count, leucocytes ratios, levels of inflammatory markers ( $C$ reactive protein- CRP, fibrinogen, and erythrocyte sedimentation rateESR), and liver enzymes (aspartate aminotransferase- AST, alanine aminotransferase- ALT, creatine kinase- CK, and gamma-glutamyl transferase- GGT) were determined in all respondents.

\section{RESULTS}

The concentrations of CRP, fibrinogen, ESR, platelet-lymphocyte ratio and monocytelymphocyte ratio in subjects with PTSD were statistically significantly higher than those in the control group. Levels of AST and GGT in PTSD subjects were statistically significantly higher than of those in the control group subjects. Statistically significant positive correlation was found between serum AST and CRP concentration (Rho = 0.416; $\mathrm{P}=0.022$ ), as well as GGT and CRP concentration (Rho $=0.395 ; \mathrm{P}=0.031$ ).

\section{CONCLUSIONS}

Results indicate the relationship between liver pathology and inflammation in the complex pathogenesis of PTSD. These can be used in future researches and development of a new diagnostic approach and treatment that may lead to a longer lifespan of PTSD patients.

\section{KEY WORDS}

PTSD, Inflammation, Liver Enzymes
Corresponding Author: Jasmin Musanovic, Department of Biology and Human Genetics, Medical Faculty, University of Sarajevo, Bosnia and Herzegovina. E-mail: jasmin.m.gen@gmail.com

DOI: $10.14260 / j e m d s / 2021 / 108$

How to Cite This Article:

Fajkic A, Musanovic J, Lepara O, et al. Correlation between inflammatory markers and liver enzymes in patients with post-traumatic stress disorder. J Evolution Med Dent Sci 2021;10(08):493-498, DOI: 10.14260/jemds/2021/108

Submission 20-10-2020,

Peer Review 28-12-2020,

Acceptance 04-01-2021,

Published 22-02-2021.

Copyright (c) 2021 Almir Fajkic et al. This is an open access article distributed under Creative Commons Attribution License [Attribution 4.0 International (CC BY 4.0)] 


\section{BACKGROUND}

Posttraumatic stress disorder is characterised by a diverse collection of parameters, including intrusive reminders, perceptions of panic, emotional pain, hypervigilance, and repetitive shocking reactions that grow and continue following trauma exposure. ${ }^{1}$ In the last few decades, the interest of professionals in psychological war trauma has constantly been growing. The existence of wars in different parts of the world enables daily research into the consequences of psychological war trauma, both among soldiers and among the population living in war-torn territories or their immediate vicinity.

PTSD is known to be included in various pathophysiological processes at neural, metabolic, inflammatory, and organic levels. Studies of the neurobiology of PTSD mainly focus on two biological systems: the hypothalamus-pituitary-adrenal axis (HPA) in connection to the immune system with the aim to establish their role in the pathogenesis of PTSD systemic consequences..$^{2,3}$

Intense stress is accompanied by the release of endogenous stress hormones (cortisol, adrenaline and noradrenaline, vasopressin, oxytocin, and endogenous opioids). In the case of prolonged chronic stress, there is a dysfunction of this stress response system and its desensitisation. Immunoreactivity studies in the PTSD model are not yet consistent and are relatively scarce compared to those in neurobiology or immunological studies in depressive disorders, and published papers that simultaneously monitor hormonal and immune parameters in the same subjects are very rare. Despite confirmation of the role of inflammation in PTSD, contradictory findings have also been reported, with some research showing no correlation between inflammation and PTSD symptoms or decreased inflammatory markers in individuals with PTSD. ${ }^{4-6}$

More and more research show that the consequences of this syndrome can be observed in numerous organs and organ systems. In recent years, as understanding the role of stress mediators has improved, data on the effects of stress on the onset and development of liver damage during acute and chronic liver disease have taken on a new dimension. For a long time, numerous researchers have suggested that stress affects the haemodynamics of hepatic blood flow by inducing vasospasm and centrilobular hypoxia, leading to liver damage. Psychosocial stress alone can lead to significant inflammatory and fibrosing changes in liver tissue affected by the cirrhosis process. $^{7}$

Considering the growing number of data that PTSD is not just a disease in the field of psychiatric diseases, and new data on complex pathogenesis, it is necessary to establish the interaction of numerous factors that contribute to the characteristic picture and development of PTSD. According to the proven role of the inflammatory process, as a possible potential basis of the disease itself and the involvement of other organs, due to the activation of the HPA axis, the question arises whether these processes are interconnected, i.e., whether there is a connection between inflammatory indicators and indicators of impaired liver function in patients with this disease. We wanted to examine the relationship between inflammatory changes and liver enzyme concentrations in patients with chronic PTSD to analyse possible connection between HPA-axis, immune system and peripheral organ dysfunction.

\section{METHODS}

The cross-sectional study included 46 consecutive male patients hospitalised at the Department of Psychiatry, Medical Centres, Bosnia and Herzegovina over a period of 24 months (March 2017 - March 2019), with a diagnosis of PTSD, according to the criteria of DSM V and ICD 10. Bosnia and Herzegovina don't have statistic evidence about the total number of war veterans with PTSD. That is why our sample is made up by snowball method with Bosnian and Herzegovinian population and with a very low statistical and data base evidence. They were evaluated with the Mini International Neuropsychiatric Interview, Brief Symptom Inventory, and the Mississippi Scale by an experienced psychiatrist. Based on the exclusion criteria, 16 patients were excluded from the study because of chronic alcohol consumption $(\mathrm{N}=8)$, inflammatory disease $(\mathrm{N}=3)$, use of drugs that affect liver function $(\mathrm{N}=4)$, and the inability to obtain an adequate blood sample $(\mathrm{N}=1)$. Laboratory tests were performed for the remaining patients $(\mathrm{N}=30)$.

The control group consisted of 30 healthy males, randomly selected volunteers without the experience of war and with no known medical disease. All participants had no familiar history of any other disease or disorders especially psychiatric and liver disorders. Inclusion and exclusion criteria were followed for this group as well.

Written informed consent for inclusion in the study was obtained from all the patients and healthy controls. The study was carried out by the Declaration of Helsinki, as revised in 2000. The ethical committee of the clinical centre university of Sarajevo approved this study.

\section{Inclusion Criteria}

1. PTSD male war veterans aged 40 years and over.

2. Control group of male individuals with no history of any disorder and without war experience

3. Signed informed consent.

\section{Exclusion Criteria}

1. Diagnosis of systemic inflammatory and septic conditions, malignant diseases, liver diseases and other psychiatric comorbidities.

2. Chronic alcohol consumption

3. Treatment with drugs that impair liver function.

\begin{abstract}
Measures
Blood samples were taken from all participants, by cubital vein puncture, for the following laboratory analyses: leukocytes, platelets, differential blood count, fibrinogen, C-reactive protein, erythrocyte sedimentation rate, and liver enzymes (AST, ALT, CK, and GGT). Leukocyte count, differential blood count, and platelet count were determined using an automatic Beckman Coulter STKS haematology analyser. Based on the obtained values, the following inflammatory ratios were calculated: neutrophil / lymphocyte ratio, platelet / lymphocyte ratio, and monocyte / lymphocyte ratio.
\end{abstract}


The concentration of CRP in the serum of the subjects was determined by laser nephelometry (BN II analyser) as reference values of CRP in serum using this method are taken from 0 to $5 \mathrm{mg} / \mathrm{L}$. Fibrinogen was determined by the coagulation method on a coagulometer from Amelung with Roche reagent. Reference values for plasma fibrinogen concentration for this laboratory were determined in subjects older than 30 years and are $1.8-3.8 \mathrm{~g} / \mathrm{L}$. Serum concentrations of the enzymes were determined spectrophotometrically using an automatic biochemical analyser Selectra. Reference values: AST - from 0 to $31 \mathrm{U} / \mathrm{l}$, ALT - from 0 to $36 \mathrm{U} / \mathrm{l}, \mathrm{CK}$ - from 0 to $175 \mathrm{U} / \mathrm{l}$, GGT - from 9 to $35 \mathrm{U} / \mathrm{l}$.

\section{Statistical Analysis}

The results were processed by standard statistical methods using the SPSS computer program for statistical analysis (SPSS Statistical Package for Social Sciences) version 21.0 (Chicago, IL, USA). The Shapiro-Wilk test, considering the sample size, was used to assess the normality of the distribution of continuous variables. Results are expressed as mean $(\bar{X})$ and standard deviation (SD) for continuous independent variables that followed normal distribution, and median and interquartile range for independent continuous variables that did not follow normal distribution.

The significance of the difference for the continuous independent variables that followed the normal distribution was tested by Student t-test. The significance of the difference for independent continuous variables that did not follow the normal distribution was tested by the Mann-Whitney test for independent samples. The correlation coefficient was determined by the Pearson method in the case when there was a linear relationship between the observed variables and a continuous normal distribution, or by the Spearman method when this condition was not satisfied. Values of $\mathrm{P}<0.05$ were considered statistically significant.

\section{RESULTS}

\begin{tabular}{|c|c|c|c|}
\hline Variables & PTSDG $(\mathrm{N}=30)$ & CG $(N=30)$ & $\mathbf{P}$ \\
\hline Leukocytes $\left(10^{9} / \mathrm{L}\right)$ & $7.2(6.2-7.6)$ & $6.9(5.9-8.3)$ & 0.663 \\
\hline Neutrophils $\left(10^{9} / \mathrm{L}\right)$ & $4.07 \pm 1.04$ & $4.11 \pm 0.97$ & 0.861 \\
\hline Basophils $\left(10^{9} / \mathrm{L}\right)$ & $0.06 \pm 0.02$ & $0.07 \pm 0.02$ & 0.109 \\
\hline Eosinophils $\left(10^{9} / \mathrm{L}\right)$ & $0.142 \pm 0.034$ & $0.148 \pm 0.03$ & 0.211 \\
\hline Lymphocytes $\left(10^{9} / \mathrm{L}\right)$ & $2.5(2.3-2.8)$ & $2.3(1.8-2.7)$ & 0.074 \\
\hline Monocytes $\left(10^{9} / \mathrm{L}\right)$ & $0.37 \pm 0.13$ & $0.34 \pm 0.09$ & 0.324 \\
\hline Platelets $\left(10^{9} / \mathrm{L}\right)$ & $291.8 \pm 41.8$ & $258.0 \pm 51.0$ & $0.007^{*}$ \\
\hline NLR & $1.8 \pm 0.3$ & $1.6 \pm 0.4$ & $0.006^{*}$ \\
\hline PLR & $120.3(109.2-153.1)$ & $98.3(78.5$ - 125.7) & $0.001^{*}$ \\
\hline MLR & $0.14(0.1-0.2)$ & $0.10(0.1-0.2)$ & 0.988 \\
\hline CRP (mg / L) & $5.8(4.8-6.9)$ & $1.4(0.8-2.4)$ & $<0.001^{*}$ \\
\hline Fibrinogen $(\mathrm{g} / \mathrm{L})$ & $4.2(3.9-4.9)$ & $2.8(2.4-3.4)$ & $<0.001^{*}$ \\
\hline $\operatorname{ESR}(\mathrm{mm} / \mathrm{h})$ & $6.2 \pm 3.4$ & $5.7 \pm 2.9$ & 0.077 \\
\hline $\operatorname{AST}(\mathrm{U} / \mathrm{L})$ & $21.0 \pm 11.0$ & $13.5 \pm 7.1$ & $0.003^{*}$ \\
\hline $\operatorname{ALT}(\mathrm{U} / \mathrm{L})$ & $21.5(19.8-32.3)$ & $19.0(15.8-28.3)$ & 0.06 \\
\hline $\mathrm{CK}(\mathrm{U} / \mathrm{L})$ & $90.0(74.5-135.0)$ & $84.5(58.5-109.0)$ & 0.206 \\
\hline GGT (U / L) & $29.5(20.0-44.3)$ & $19.5(13.5-28.3)$ & $0.001^{*}$ \\
\hline \multicolumn{4}{|c|}{$\begin{array}{l}\text { Table 1. Difference in WBC Counts, Inflammatory } \\
\text { Markers and Liver Enzymes between Experimental } \\
\text { Group of Veterans with PTDS and Control Group }\end{array}$} \\
\hline \multicolumn{4}{|c|}{ *Statistically significant at level of 0.05} \\
\hline \multicolumn{4}{|c|}{$\begin{array}{l}\text { NLR - neutrophil-lymphocyte ratio; PLR- platelet-lymphocyte ratio; MLR } \\
\text { monocyte-lymphocyte ratio; CRP - C reactive protein; AST - aspartate } \\
\text { aminotransferase; ALT - alanine aminotransferase; CK - cretin kinase; } \\
\text { GGT - gamma-glutamyl transferase; PTSDG - patients with PTSD; CG - } \\
\text { control group; P - probability }\end{array}$} \\
\hline
\end{tabular}

Table 1 shows the difference in WBC counts, inflammatory markers and liver enzymes between experimental group of veterans with PTDS and control group.

As the results showed, we found in the group of PTSDs veterans', significant higher value of platelet count $(P=0.007)$, NLR ( $\mathrm{P}=0.006)$, PLR $(\mathrm{P}=0.001)$, CRP $(\mathrm{P}<0.001)$, fibrinogen $(\mathrm{P}<0.001)$, AST $(\mathrm{P}=0.003)$ and GGT $(\mathrm{P}=0.001)$, in comparison to control group of healthy volunteers.

Correlations between haematological parameters and levels of liver enzymes in the group of veterans with PTSD are presented in Table 2. A statistically significant negative correlation was found between the serum ALT concentration with WBC count (Rho $=-0.420 ; \mathrm{P}=0.021$ ) and neutrophils (Rho $=-0.434 ; \mathrm{P}=0.017$ ), as well as a statistically significant positive correlation between serum GGT concentration and peripheral blood lymphocyte count $(\mathrm{Rho}=0.400 ; \mathrm{P}=0.028)$. Other associations of liver enzymes with haematological parameters in the group of veterans with PTSD did not reach the level of statistical significance.

\begin{tabular}{|c|c|c|c|c|}
\hline \multirow{2}{*}{$\begin{array}{l}\text { Haematological } \\
\text { Parameters }\end{array}$} & \multicolumn{4}{|c|}{ Liver Enzymes } \\
\hline & $\begin{array}{c}\text { AST } \\
(\mathbf{U} / \mathrm{L})\end{array}$ & $\begin{array}{c}\text { ALT } \\
(\mathrm{U} / \mathrm{L})\end{array}$ & $\begin{array}{c}\text { CK } \\
(\mathrm{U} / \mathrm{L})\end{array}$ & $\begin{array}{c}\text { GGT } \\
(\mathbf{U} / \mathrm{L})\end{array}$ \\
\hline WBC (x $\left.10^{9} / \mathrm{L}\right)$ & Rho $=-0.144$ & Rho $=-0.420^{*}$ & Rho $=-0.184$ & Rho $=0.131$ \\
\hline Neutrophils (x 109 / L) & $R=-0$. & Rho $=-0$ & 198 & .036 \\
\hline Basophils (x $\left.10^{9} / \mathrm{L}\right)$ & $R=0.022$ & Rho $=-0.054$ & Rho $=-0.147$ & Rho $=-0.029$ \\
\hline Eosinophils (x 109 / L) & $\mathrm{R}=0$ & Rho $=0.019$ & Rho $=-0.126$ & Rho $=0.083$ \\
\hline Lymphocytes (x 1099/L) & Rho $=0.046$ & Rho $=-0.157$ & Rho $=0.029$ & Rho $=0.400^{*}$ \\
\hline Monocytes (x 109/L) & $\mathrm{R}=0.231$ & Rho $=0.109$ & Rho $=-0.193$ & Rho $=0.145$ \\
\hline Platelets (x $\left.10^{9} / \mathrm{L}\right)$ & $\mathrm{R}=0.247$ & Rho $=0.323$ & Rho $=0.186$ & Rho $=0.164$ \\
\hline \multicolumn{5}{|c|}{$\begin{array}{l}\text { Table 2. Correlation of Haematological Parameters } \\
\text { and Liver Enzymes in PTSD Patients }\end{array}$} \\
\hline \multicolumn{5}{|c|}{ *Statistically significant at level of 0.05} \\
\hline \multicolumn{5}{|c|}{$\begin{array}{l}\text { AST - aspartate aminotransferase; ALT - alanine aminotransferase; CK - } \\
\text { cretin kinase; GGT - gamma-glutamyl transferase; P - probability; Rho - } \\
\text { Spearman correlation coefficient; r - Pearson correlation coefficient }\end{array}$} \\
\hline
\end{tabular}

\begin{tabular}{|ccccc|}
\hline $\begin{array}{c}\text { Inflammatory } \\
\text { Ratios }\end{array}$ & AST & ALT & CK & GGT \\
(U / L) & (U / L) & (U / L) & (U / L) \\
PLR & $\mathrm{r}=0.171$ & $\mathrm{Rho}=0.152$ & $\mathrm{Rho}=-0.185$ & $\mathrm{Rho}=0.165$ \\
MLR & $\mathrm{Rho}=0.295$ & $\mathrm{Rho}=0.381^{*}$ & $\mathrm{Rho}=0.102$ & $\mathrm{Rho}=0.170$ \\
\hline Table 3. Correlation between Inflammatory Ratios \\
and Liver Enzymes in PTSD Patients \\
\hline *Statistically significant at level of 0.05 \\
\hline NLR - neutrophil-lymphocyte ratio; PLR - platelet-lymphocyte ratio; MLR \\
- monocyte-lymphocyte ratio; AST - aspartate aminotransferase; ALT - \\
alanine aminotransferase; CK - cretin kinase; GGT - gamma-glutamyl \\
transferase; P - probability; Rho - Spearman correlation coefficient; r - \\
Pearson correlation coefficient \\
\hline
\end{tabular}

\begin{tabular}{|c|c|c|c|c|}
\hline \multirow{2}{*}{$\begin{array}{l}\text { Inflammatory } \\
\text { Markers }\end{array}$} & \multicolumn{4}{|c|}{ Liver Enzymes } \\
\hline & $\begin{array}{l}\text { AST } \\
(U / L)\end{array}$ & $\begin{array}{l}\text { ALT } \\
(U / L)\end{array}$ & $\begin{array}{c}\text { CK } \\
(U / L)\end{array}$ & $\begin{array}{l}\text { GGT } \\
(U / L)\end{array}$ \\
\hline CRP (mg / L) & Rho $=0.416^{*}$ & Rho $=0.209$ & Rho $=0.160$ & Rho $=0.395^{*}$ \\
\hline Fibrinogen $(\mathrm{g} / \mathrm{L})$ & Rho $=-0.317$ & Rho $=0.076$ & Rho $=0.113$ & Rho $=0.224$ \\
\hline $\operatorname{ESR}(\mathrm{mm} / \mathrm{h})$ & $R=-0.104$ & Rho $=-0.047$ & Rho $=-0.166$ & Rho $=0.059$ \\
\hline \multicolumn{5}{|c|}{$\begin{array}{l}\text { Table 4. Correlation between Inflammatory Markers } \\
\text { and Liver Enzymes in PTSD Patients }\end{array}$} \\
\hline \multicolumn{5}{|c|}{ *Statistically significant at level of 0.05} \\
\hline \multicolumn{5}{|c|}{$\begin{array}{l}\text { AST - aspartate aminotransferase; ALT - alanine aminotransferase; CK - } \\
\text { cretin kinase; GGT - gamma-glutamyl transferase; CRP - C reactive } \\
\text { protein; ESR - erythrocyte sedimentation rate; P - probability; Rho - } \\
\text { Spearman correlation coefficient; r - Pearson correlation coefficient }\end{array}$} \\
\hline
\end{tabular}

A statistically significant positive association between serum ALT concentration and platelet / lymphocyte ratio was found in the group of veterans with PTSD $(\mathrm{Rho}=0.381 ; \mathrm{P}=$ 0.038). Other associations between liver enzymes and 
haematological relationships were not statistically significant (Table 3).

Correlation analysis indicated a significant positive relationship between serum AST concentration and serum CRP concentration ( $R h o=0.416 ; \mathrm{P}=0.022$ ), as well as between serum GGT concentration and serum CRP concentration (Rho $=0.395 ; \mathrm{P}=0.031)($ Table 4$)$.

\section{DISCUSSION}

According to the available literature, this is the first study to examine the association between inflammatory markers and liver enzyme levels in Bosnian patients with chronic PTSD. What should be emphasised is that researchers have mainly analysed the association of data of these two types of markers in acute PTSD condition.

The connection between psychological distress and somatic symptoms has been observed and described since ancient times. New, exact research confirms, otherwise, very old observations, that there is a close connection between stress and the development of several diseases.

Recent research has shown that the underlying psychobiological pathways linking PTSD to an increased risk of chronic disease are altered autonomic nervous system function and impaired hypothalamic-pituitary-adrenal axis response to perceived stress. Previous research has reported that the pathogenesis of PTSD shows a combined occurrence of immune dysregulation and disorders of neurotransmitter systems in brain tissue. This results in the development of lowgrade chronic inflammation, one of the main features of this disease. ${ }^{8}$ The origin of this low-grade inflammation is still not sufficiently elucidated. Some authors have suggested that PTSD leads to the development of an inflammatory condition due to dysregulation in neuroendocrine pathways, including the autonomic nervous system-hypothalamus-pituitary axis. ${ }^{9}$ In that sense, analysing the change in the concentrations of inflammatory markers is necessary to confirm this hypothesis.

After the analysis of inflammatory markers levels in the group of PTSD veterans, we found significant higher value of platelet count, NLR, PLR, CRP, and fibrinogen compared to control group of healthy volunteers.

Although we did not find data from platelet count analysis in PTSD patients, platelets' significant potential role in developing psychiatric illness should be emphasised here. Platelet activation and changes in the normal process of haemostasis and the inflammatory process play an important role in psychiatric disorders. Their role is based on releasing serotonin, which has a significant place in the pathophysiology of depression and psychiatric disorders, and the release of numerous pro-inflammatory molecules, such as IL-1, metalloprotein TF, P-selectin, and CD40L.10,11

For this reason, platelets are becoming more and more interesting to researchers in the field of psychiatry due to their possible role, not only as a marker but also as a potential cofactor in the pathogenesis of neuropsychiatric disorders. It is thought that platelets, with their cellular molecules, could be one of the main links between acute and chronic psychiatric or organic stress-related disorders. ${ }^{12}$

The inflammatory ratios' calculation is a new, easily measured, and inexpensive choice, suitable for routine use to demonstrate inflammation. They are advantageous compared to other biomarkers because of the low cost, routine use, general clinical conditions, and high inter-laboratory repeatability, given that the ratios are derived from the WBCs count. These ratios integrate information on innate and acquired immunity and represent a reliable and easily accessible measure of the inflammatory condition for better understanding the pathogenesis of various disease.13 The results of several studies have shown that inflammatory ratios can be used not only for diagnostic and prognostic purposes but also to be the main and most accurate indicators of the intensity of the inflammatory process in various pathophysiological disorders (cardiovascular diseases, malignant tumours, autoimmune diseases, etc.).14,15

As interest in inflammatory disorders has grown as potential mechanisms in the development of psychiatric illness, there is a need to use inflammatory ratios, NLP, PLR, and MLR, as simpler diagnostic tools to assess the state of inflammation neuropsychiatric diseases. Increased NLR, PLR, and MLR have been found in patients with bipolar disorders, schizophrenia, depression and Alzheimer disease. ${ }^{13}$

We found higher CRP values in veterans with PTSD compared to healthy subjects. The results obtained in our study are in line with the results of previous studies, which found that in PTSD patients there is a significantly higher possibility of an increase in serum CRP compared to healthy subjects. ${ }^{16,17}$ The authors of these studies believe that there is a strong association between the development of PTSD and low-grade inflammation, which together cause a weakening of physical health and the development of various comorbidities.

The results of previous research aimed at confirming proinflammatory activity in the development of PTSD are not consistent; some studies have reported reduced levels of inflammatory markers, such as proinflammatory interleukins, TNF- $\alpha$, and CRP, in patients with PTSD compared with healthy controls. Although elevated levels of TNF- $\alpha$ and IL-1 $\beta$ have been found in patients with PTSD, Von Känel et al. did not find a statistically significant difference in serum CRP concentration in patients with PTSD than the control group.

A longitudinal research, however, recently discovered that elevated baseline CRP was an important predictor of PTSD prior to deployment, indicating that elevated inflammation is a risk factor for PTSD. ${ }^{18}$

Previous research has shown that chronic psychological stress leads to liver damage in humans. Stress caused by illness e.g., patients after myocardial infarction, leads to increased levels of liver enzymes. ALT, AST, and ALP are enzymes found in the body and are most commonly increased in liver disease or injury. Elevated ALT and AST levels are characteristic of the hepatocellular disease, while increased ALP is characteristic of hepatobiliary disease. A small number of studies have studied the relationship between stress and liver enzyme levels in humans.

Kaji et al. have shown that serum transaminase levels increase due to stress in rats in which galactosamine produced liver damage. The authors suggest that stress is caused by HPA-activated pathways associated with inflammation. ${ }^{19}$

Experimental research has shown that in rats, psychological stress leads to liver tissue damage, manifested by an increase in liver transaminases and alkaline phosphatase in the circulation. Von Kannel et al. investigated the hypothesis that the severity of PTSD after myocardial 
infarction will impact liver enzyme levels. The authors conclude that PTSD, which develops after acute myocardial infarction, is a significant indicator of ALT and ALP enzymes' plasma levels. Liver enzyme levels are estimated to remain elevated for a period of two years after the diagnosis of PTSD. Based on the previous finding, it can be concluded that the duration of PTSD has a greater impact than the severity of the disease itself when it comes to liver enzymes. ${ }^{20}$

The explanation for this phenomenon is that PTSD can provoke liver inflammation through the action of catecholamines and glucocorticoids, which are peripheral effectors of the stress response. Noradrenergic dysregulation affects the initiation and maintenance of recurrent PTSD symptoms. Concerning catecholaminergic hyperactivity of PTSD, patients show hypocorticism resulting from dysregulation of the HPA axis. The immunosuppressive action of glucocorticoids is based on the inhibition of liver macrophages to release proinflammatory cytokines. For this reason, it is believed that reduced glucocorticoid secretion, combined with psychological stress, can lead to increased release of proinflammatory interleukins, IL-1 and IL-6, and TNF- $\alpha$ by macrophages. The increased sympathetic effect on the liver and hypocorticism provide reliable mechanisms that can potentially induce elevated liver enzyme levels in patients with myocardial infarction with more severe PTSD.21 In addition, recent studies have identified additional mechanisms linking stress and liver dysfunction (hypoxiareoxygenation, over-activation of Kupffer cells and oxidative stress, and influx of gut-derived lipopolysaccharide (LPS) and norepinephrine). ${ }^{21}$

The relationship between serum liver enzyme levels and inflammatory markers in PTSD has not been adequately analysed to date. The results of our study found a statistically significant correlation between different inflammatory markers (WBC number, CRP lymphocyte count, PLR) and liver enzymes (AST, GGT) in veterans with PTSD. As we could not find similar research in the available literature, we could not compare and analyse our results to support our findings.

What should be singled out as a significant result is the connection between GGT and CRP levels, which could be evidence of the common role of the inflammatory process and oxidative stress in the development of PTSD, since GGT is one of the markers of oxidative stress. One potential cause for associating PTSD, oxidative stress and liver damage is by persistent and repetitive activation of the hypothalamicpituitary-adrenal axis that develops during trauma reexperience. Increased toxicity in response to stressors leads to the activation of oxidative stress reactions, resulting in an increase in reactive oxygen species and / or nitrogen concentration. These reactive molecules lead to modification of the structural phospholipids of neuronal membranes through a peroxidation mechanism, thereby affecting neuronal functions, such as signal transduction. ${ }^{22}$

\section{CONCLUSIONS}

In recent years, stress has been identified as an important factor in the pathogenesis and progression of several important liver disorders. Stress affects the immune system and numerous mechanisms within and between cells. A better understanding of how stress alters the liver's inflammatory process will provide additional opportunities to manage important liver diseases from a clinical perspective. This would affect patients' quality of life by shortening hospitalisation and ensuring the correct therapeutic approach. Results of the current study indicate the potential involvement of low-grade inflammation with liver dysfunction in PTSD. To validate our findings, further major prospective populationbased studies are required. Extensive clinical trials can be developed to test positive correlations between elevated levels of inflammatory parameters and several serological and imaging parameters used to assess the degree of liver disease in patients with PTSD.

\section{Limitations}

There are several limitations to the current study. Our participants were only male veterans from one unit of traumarelated disabilities, and our findings may not be generalisable to females with PTSD or to individuals with other, noncombatrelated PTSD. Also, the study's cross-sectional design prevents us from deducting any causal links between our results.

Data sharing statement provided by the authors is available with the full text of this article at jemds.com.

Financial or other competing interests: None.

Disclosure forms provided by the authors are available with the full text of this article at jemds.com.

A.F., O.L., and J.M. gave substantial contribution to the conception or design of the work and in the acquisition, analysis and interpretation of data for the work. Each author had role in drafting the work and revising it critically for important intellectual content and gave final approval.

\section{REFERENCES}

[1] American Psychiatric Association. Diagnostic and statistical manual of mental disorders. $5^{\text {th }}$ edn. Washington, DC: American Psychiatric Association 2013.

[2] Girgenti MJ, Hare BD, Ghosal S, et al. Molecular and cellular effects of traumatic stress: implications for PTSD. Curr Psychiatry Rep 2017;19(11):85.

[3] Neigh GN, Ali FF. Co-morbidity of PTSD and immune system dysfunction: opportunities for treatment. Curr Opin Pharmacol 2016;29:104-10.

[4] Söndergaard HP, Hansson LO, Theorell T. The inflammatory markers C-reactive protein and serum amyloid $\mathrm{A}$ in refugees with and without posttraumatic stress disorder. Clin Chim Acta 2004;342(1-2):93-8.

[5] Sutherland AG, Cameron GA, Alexander DA, et al. Proinflammatory markers in prediction of posttraumatic psychological symptoms: a prospective cohort study. Mediators Inflamm 2008;2008:640659.

[6] von Känel R, Hepp U, Kraemer B, et al. Evidence for lowgrade systemic proinflammatory activity in patients with posttraumatic stress disorder. J Psychiatr Res 2007;41(9):744-52.

[7] Vere CC, Streba CT, Streba LM, et al. Psychosocial stress and liver disease status. World J Gastroenterol 2009;15(24):2980-6.

[8] Speer K, Upton D, Semple S, et al. Systemic low-grade inflammation in post-traumatic stress disorder: a systematic review. J Inflamm Res 2018;11:111-21. 
[9] Neigh GN, Ali FF. Co-morbidity of PTSD and immune system dysfunction: opportunities for treatment. Curr Opin Pharmacol 2016;29:104-10.

[10] Dietrich-Muszalska A, Wachowicz B. Platelet haemostatic function in psychiatric disorders: effects of antidepressants and antipsychotic drugs. World J Biol Psychiatry 2017;18(8):564-74.

[11] Wachowicz B. Blood platelet as a peripheral cell in oxidative stress in psychiatric disorders. In: DietrichMuszalska A, Chauhan V, Grignon S, eds. Studies on psychiatric disorders. oxidative stress in applied basic research and clinical practice. New York, NY: Human Press 2015.

[12] Koudouovoh-Tripp P, Sperner-Unterweger B. Influence of mental stress on platelet bioactivity. World J Psychiatry 2012;2(6):134-47.

[13] Mazza MG, Rossetti A, Clerici M. A review of neutrophillymphocyte, monocyte-lymphocyte and platelet lymphocyte ratios use in psychiatric disorders. World Journal of Depression Anxiety 2018;1(1):1002.

[14] Afari ME, Bhat T. Neutrophil to lymphocyte ratio (NLR) and cardiovascular diseases: an update. Expert Rev Cardiovasc Ther 2016;14(5):573-7.

[15] Chandrashekara S, Ahmad MM, Renuka P, et al. Characterization of neutrophil-to-lymphocyte ratio as a measure of inflammation in rheumatoid arthritis. Int J Rheum Dis 2017;20(10):1457-67.
[16] Spitzer C, Barnow S, Völzke H, et al. Association of posttraumatic stress disorder with low-grade elevation of C-reactive protein: evidence from the general population. J Psychiatr Res 2010;44(1):15-21.

[17] Vaccarino V, Bremner JD, Afzal N, et al. Posttraumatic stress disorder is associated with higher C-reactive protein levels. J Am Coll Cardiol 2010;55(10A):A176.

[18] Eraly SA, Nievergelt CM, Maihofer AX, et al. Assessment of plasma C-reactive protein as a biomarker of posttraumatic stress disorder risk. JAMA Psychiatry 2014;71(4):423-31.

[19] Kaji I, Sekiya C, NamikI M. Psychosomatic study of the patients with liver disorders: including an experimental study. Jap J psychosom Med 1981;21:302-12.

[20] von Känel R, Abbas CC, Begré S, et al. Association between posttraumatic stress disorder following myocardial infarction and liver enzyme levels: a prospective study. Dig Dis Sci 2010;55(9):2614-23.

[21] Joung JY, Cho JH, Kim YH, et al. A literature review for the mechanisms of stress-induced liver injury. Brain Behav 2019;9(3):e01235.

[22] Miller MW, Lin AP, Wolf EJ, et al. Oxidative stress, inflammation and neuroprogression in chronic PTSD. Harv Rev Psychiatry 2018;26(2):57-69. 